\title{
Littérature, culture nationale et guerre
}

\author{
Alfonso Reyes et les politiques de l'humanisme \\ au Mexique (1939-1945) ${ }^{1}$
}

Résumé: Cet article propose une lecture politique du livre d'Alfonso Reyes, La crítica en la edad ateniense (1941). Écrit en pleine Seconde Guerre mondiale, ce livre, qui a reçu le premier Prix national de littérature au Mexique, propose une actualisation de la culture grecque classique et suggère, en même temps, un projet de modélisation éthique pour le citoyen en temps de crise et de guerre. Avec ce livre, Reyes a répondu à une série de préoccupations concernant le rôle de la littérature et de l'intellectuel dans la formation de la polis nationale et mondiale de l'époque.

Mots-clés: universalisme; humanisme; hellénisme; premier Prix National de Littérature au Mexique; Institut International de Coopération Intellectuelle; Alfonso Reyes

\section{Le prix}

En décembre 1945, dans le cadre de la création des Prix Nationaux des Sciences et des Arts, le gouvernement mexicain décerne le premier Prix National de Littérature [Premio Nacional de Literatura]. La création de cette récompense fait partie d'un processus intégral de consolidation des institutions de l'état national postrévolutionnaire. À l'issue d'une longue période de conflit entre 1910 et 1919, le Mexique commence à expérimenter, à partir des années trente, une étape de stabilité relative qui se manifeste par la fondation d'institutions et l'impulsion de réformes sociales, éducatives et économiques. Durant la période de 1934 à 1940, sous la présidence du général Lázaro Cárdenas, le pays connaît un des moments de plus grande attente sociale: grâce à une réforme agraire, des terres sont distribuées aux paysans; l'éducation est déclarée socialiste; l'industrie pétrolière est nationalisée; de nombreuses instances de défense des droits sociaux et culturels

1 Traduit de l'espagnol par Hrindanaxi G. Villagómez et Sergio Ugalde Quintana. Une version étendue de cet article sera publiée dans Ugalde Quintana et Ottmar Ette (à paraître).

Sergio Ugalde Quintana, El Colegio de México

2 Open Access. (c) 2021 Sergio Ugalde Quintana (cc) BY-NC-ND This work is licensed under a Creative Commons Attribution-NonCommercial-No-Derivatives 4.0 License.

https://doi.org/10.1515/9783110691504-007 
sont créées (Pérez Monfort 2019). Dans le même esprit, sous la présidence suivante, celle de Manuel Ávila Camacho, de 1940 à 1946, certaines des principales institutions culturelles du pays sont créées: en 1940, El Colegio de México a été inauguré; en 1943, El Colegio Nacional; en 1946, l'Instituto Nacional de Bellas Artes. Enfin, durant cette période toute une structure d'état est fondée à partir de nouveaux organismes et institutions. La création en 1945 du Prix National de Littérature, sous l'égide des structures gouvernementales, fait partie de ce vaste processus. Dans l'exposé des motifs présenté à la Chambre des députés en septembre 1944 pour l'approbation de la loi instituant les Prix Nationaux des Sciences et des Arts, il est dit:

Le gouvernement actuel de la République s'est fondamentalement préoccupé de développer les manifestations de la culture supérieure dans le pays [...]. Il a aidé, dans la mesure de ses moyens, les écrivains et les artistes mexicains, bien que cela ait été à plusieurs reprises sous une forme dispersée [...]. Toutefois, pour le plein développement de la culture supérieure du pays, il est nécessaire que le gouvernement donne une impulsion déterminée, qui, tout en accordant un prix en argent à ceux qui le méritent pour leurs activités dans le domaine de la culture, entraîne également la plus haute distinction que la nation puisse accorder à ceux qui mettent tout en œuvre pour lui donner du prestige et l'ennoblir devant eux-mêmes et devant les autres pays du monde (Díaz Arciniega 1991, 19).

Ainsi, avec la création de ces Prix, une articulation institutionnelle entre l'État national post-révolutionnaire et la communauté artistique et scientifique du pays a commencé. Octavio Paz, en faisant référence à ce lien, a appelé l'État « l'ogre philanthropique » (1974, 38-44). Si l'on considère la valeur sociale, culturelle, politique et symbolique qui a reçu à l'origine le Prix National de Littérature, les questionnements deviennent inévitables. Qui serait le lauréat de ce nouveau rite institutionnel? Quels avaient été les critères de sélection du vainqueur? Quelle œuvre littéraire avait été reconnue à cet acte?

En regardant de près le rapport du jury crée spécialement pour cette remise de prix, plusieurs possibilités pour sélectionner un vainqueur apparaissent: entre 1940 et 1945, période concernée par la convocation, avait été publiés des romans, des poèmes, des recueils de contes pouvant satisfaire aux conditions de base du prix : il s’agissait d'œuvres «stylistiquement et formellement appropriées » et « représentatives de n’importe quel aspect du Mexique ». Dans ces années-là, plusieurs jeunes écrivains avaient écrit et publié certaines des œuvres fondamentales du $\mathrm{XX}^{\mathrm{e}}$ siècle mexicain: José Revueltas a publié son roman El luto humano [Le deuil humain]; Octavio Paz et Efraín Huerta ont publié, respectivement, les recueils de poèmes $A$ la orilla del mundo [Au bord du monde] et Los hombres del alba [Les hommes de l'aube]; Francisco Tario, a publié le recueil de contes: Aquí abajo [Ici, en bas]. D’autres écrivains, ayant déjà une trajectoire reconnue, avaient 
publié des œuvres emblématiques: Carlos Pellicer a fait connaître Recinto y otras imágenes [Recinto et autres images]; Xavier Villaurrutia, Décima Muerte; Agustín Yañez, Pasión y convalescencia [Passion et convalescence]; Mariano Azuela, Nueva burguesía [Nouvelle Bourgeoisie] ; Francisco Rojas González, La negra Angustias [La négresse Angustias]; Ermilo Abreu Gómez, Canek; Gregorio López et Fuentes, Una carta a Dios [Une lettre adressée à Dieu]. Comme on peut le constater, le scénario était pluriel et diversifié. La décision du jury n’a donc pas été facile à prendre. Précisément en raison des difficultés liées au choix d'une pièce sur une scène aussi diverse, le jury, qui était composé de José Vasconcelos, Julio Torri, Enrique González Martínez, Alejandro Quijano et José López Portillo y Weber, s'était prescrit une condition fondamentale: « étant donné qu'il ne s'agissait pas d'un concours de littérature régionale, nous étions convenus que le caractère autochtone n'était pas fondamental, puisque la culture mexicaine a le droit de faire entendre sa voix par rapport aux questions d'intérêt général humain et sur les problèmes universels » (Vasconcelos et al. 1945, 1). Ainsi, le jury formule catégoriquement un critère d'évaluation qui oppose le caractère " autochtone » à l'« universel ». Cette dichotomie fait résonner des concepts déjà mis en avant lors de précédentes polémiques littéraires dans le domaine littéraire mexicain.

Des années auparavant, en 1925, deux factions littéraires se sont vivement opposées: d'un côté, les représentants de la littérature de la révolution-romanciers et narrateurs - de l'autre, les poètes et essayistes représentants de l'avant-garde artistique. Le point central de discorde était la conception de la culture nationale de chaque groupe. D'un côté, ceux qui prônent une culture révolutionnaire; de l'autre, ceux qui soutiennent une culture cosmopolite. Les premiers se proclament «virils », les seconds sont qualifiés d'« efféminés ». Le différend a été traité dans les pages de certains des journaux à plus fort tirage du pays. Des articles des deux côtés allaient et venaient dans les rédactions de El Universal, Excélsior, El Demócrata, Revista de la Revistas, El Universal Ilustrado, La Antorcha, etc. (Díaz Arciniega 1989). Ce même débat s’est ravivé plus vigoureusement quelques années plus tard, en 1932, lorsque des écrivains nationalistes (autochtones) se sont heurtés aux cosmopolites (universels). À cette occasion, la controverse a été provoquée par un article qui remettait en question la validité de l'avant-garde artistique au Mexique. Une fois de plus, les termes utilisés ont polarisé les positions. Du moins, c'est ainsi que l'on a pu lire la confrontation. Pour de nombreux historiens de la littérature, deux groupes se confrontaient: d'une part, l'avant-garde cosmopolite, avec des œuvres littéraires universelles, et d'autre part, les écrivains révolutionnaires nationalistes, avec des œuvres autochtones (Sheridan 1999). Mise à distance, la confrontation semble manichéenne. Nous pourrions parler actuellement d'" autochtonismes internationalistes " ou de « cosmopolitismes vernaculaires» (Bhabha 2013; Sisikind 2016). Cependant, 
en 1945, l'horizon d'énonciation pour l'attribution du premier Prix National de Littérature laisse encore entrevoir un antagonisme entre l'autochtone et l'universel. Le jury d'alors fait le choix d'une figure et une œuvre très peu « régionalistes » en apparence mais qui montrent beaucoup d'« universel ». L'auteur récompensé, par qui s'amorçait dès lors un rituel significatif dans la polis littéraire mexicaine, est Alfonso Reyes. L’œuvre primée, La crítica en la edad ateniense (1941) [La critique à l'âge athénien], porte sur la Grèce classique.

\section{L'homme de lettres}

Au milieu des années quarante, Alfonso Reyes (1889-1959) incarne la figure de l'homme de lettres par excellence au Mexique et en Amérique latine. Son parcours littéraire, diplomatique et philologique est reconnu à divers endroits. L'écrivain vit à l'étranger pendant un peu plus d'un quart de siècle. En août 1913, âgé de 24 ans, il doit quitter le pays; son père (ancien ministre de Guerre et candidat à la présidence de la république) est assassiné au début de l’année en essayant de prendre d'assaut le palais national (Niemayer 1966). Reyes ne rentrerait pas au Mexique avant 1939. Il survit aux cinq premières années de son exil en Espagne grâce à ses activités philologiques et journalistiques; il intègre ensuite le service diplomatique et assure les fonctions d'ambassadeur du Mexique à Madrid, à Paris, à Buenos Aires et, finalement, à Rio de Janeiro (Garciadiego 2009). À chacun de ces postes, il établit des liens intellectuels solides et des dialogues littéraires intenses. En Espagne, il est proche d'Ortega y Gasset, Juan Ramón Jiménez et Ramón Menéndez Pidal; en France, de Paul Valéry et Adrienne Monnier; en Argentine, de Jorge Luis Borges et de Victoria Ocampo. À la fin des années trente, Alfonso Reyes était devenu la figure de l'écrivain, homme de lettres et humaniste par excellence du monde hispano-américain.

Fort de ce capital symbolique, Reyes rentre au Mexique en février 1939. Son rôle est fondamental pour fonder quelques-unes des institutions éducatives et de recherche du pays. Il crée et dirige La Casa de España, une institution qui, peu de temps après, a acquis le nom El Colegio de México - un centre de recherches en sciences humaines et sociales -, il collabore à la formation du Fondo de Cultura Económica - une des plus importantes sociétés éditoriales du monde hispanique - et fonde El Colegio Nacional, institution qui, tout comme le Collège de France, accueille des personnalités majeures des domaines des sciences et des arts du pays. À cette figure aux multiples facettes mêlant poète, narrateur, essayiste, philologue et diplomate, est décerné le premier Prix National de Littérature en 1945. Quelle œuvre était alors primée? 


\section{La critique à l'âge athénien}

Une fois installé au Mexique, éloigné des obligations diplomatiques et consacré à son œuvre la plupart du temps, Alfonso Reyes écrit une série d'ouvrages où il étudie le phénomène de la littérature. Les résultats de toutes ses préoccupations culminent avec le volume El Deslinde. Prolegómenos a la teoría literaria de 1944 (Rangel Guerrra 1989). Le Prix National de Littérature, cependant, ne lui est pas décerné pour ce traité, mais pour un livre né tandis qu'il écrivait El Deslinde. Une des questions qui tourmente Reyes lors de la rédaction de sa théorie littéraire est la suivante: quand avait débuté la critique littéraire en Occident? Cette inquiétude l'amène à reprendre une de ses passions de jeunesse: la lecture des œuvres de la Grèce classique ${ }^{2}$. C'est ainsi qu'entre septembre 1940 et janvier 1941, Alfonso Reyes écrit le livre: La critique à l'âge athénien. Dans les mois suivants, entre janvier et avril de cette dernière année, Reyes a lu son travail dans le cadre d'un cours à la Faculté de Philosophie et Lettres de l'UNAM. Le projet avait également un certain caractère pédagogique. Le principe qui structure cette étude est très clair ; Reyes souhaitait retracer la naissance et le développement de l'idée de critique littéraire au IV ${ }^{\mathrm{e}}$ siècle athénien. Ce principe organise tout le matériel du livre de presque 350 pages. Reyes commence par questionner les origines parmi les présocratiques, il continue avec Socrate, Aristophane, Platon, Isocrate, Aristote et termine par Théophraste et Ménandre. Durant ce parcours, il s'interroge sur les sources primaires, lit des études critiques, interroge les textes et en conclut que parmi les grecs, la critique littéraire a toujours suivi les valeurs religieuses, sociales et politiques. La critique esthétique ne s'est jamais construite comme une valeur en soi. D’où la conclusion formulée dans les dernières lignes de son livre:

Le peuple qui a doté l'humanité des œuvres poétiques les plus admirables, ressentait à peine le besoin de leur appliquer [...] le critère esthétique. Au moment de juger, il s'est livré au critère de la religion, de la morale, de la politique, et même du formalisme perceptif (Reyes 1941, 364).

L’accueil du livre est ambivalent. En général, les opinions sont divisées en deux groupes: d'un côté, ceux qui saluent la publication d'un travail scientifique et philologique solide; de l'autre, ceux qui regrettent l'absence de problématiques « mexicaines » dans cet ouvrage. Les philologues se démarquent dans le premier domaine. Certains soutiennent l'hypothèse de départ du livre. Werner Jaeger, le

2 Sur l'importance de l'univers grec dans la formation initiale d'Alfonso Reyes, on peut consulter les travaux de Susana Quintanilla (2002; 2008), Robert T. Conn (2002) et Sergio Ugalde Quintana (2019). 
philologue allemand, auteur de l'ouvrage Paideia (1933) et fondateur des études classiques à l'Université de Harvard, exprime dans une lettre personnelle à Reyes, datée du 28 mars 1942, son soutien de l'idée qu'il manque une critique esthétique à Athènes au IV ${ }^{\mathrm{e}}$ siècle.

I think it very fortunate that you have set forth with so much clarity and decision the fact that literary criticism in our sense is absent from the earlier and classical periods of Greek culture and the criticism which is uttered in those centuries with regard to what we would call literary subjects springs from other motives than a purely aesthetic appreciation [...]. It is in my opinion the greatest merit of your book that it does not discard the classical period for this reason as is often done by those interested in the problem of literary criticism in its pure form, but pursues carefully the gradual development of the critical element in Greek life and literature in all its aspects. In this way you have succeded in showing clearly how along with the moral, political and religious criticism in the classical period the critique of the aesthetic qualities of literary works gradually emerges. [...] In your "anachronistic" chapter at the end of the book you have expressed the natural reaction of the modern mind with regard to the absence of pure literary judgment from the earliest and classical periods of Greece. It is indeed not easy to see how we could return in our days to the Greek subordination of the aesthetic factor to what they thought to be the really essential moral and political factors in the poetical creations which we love. On the other hand it is about time to realize and consider earnestly the facts which you have set forth with so much vigor and in a forcible language with regard to their importance for our historical understanding of the true nature and structure of the classical Greek spirit. The result of your book with which I agree and what I have tried to say about the same problem from the opposite point of view, that of Paideia, seems to reopen the discussion of our relationship to the classical and Hellenistic forms of Greek culture (Ugalde 2009, 100-101).

Ce passage de la lettre de Jaeger souligne non seulement les mérites du livre d'Alfonso Reyes, avec lesquels le philologue allemand est d'accord, mais aussi le problème central de la philologie classique au début du $20^{\mathrm{e}}$ siècle : à savoir, quelle était la relation de l'époque actuelle avec les formes de la culture grecque classique? En d'autres termes, comment les intellectuels du $\mathrm{XX}^{\mathrm{e}}$ siècle peuvent-ils gérer le patrimoine culturel de l'Antiquité? Cependant, tous les spécialistes ne partageaient pas les conclusions d'Alfonso Reyes. Certains ont mis en doute ses prémisses. Ce fut le cas d'Ingemar Düring, le classiciste suédois, qui a déclaré que l'écrivain mexicain aurait pu nuancer ses affirmations. Pour Düring, une critique esthétique a effectivement existé dans la Grèce classique.

Si nous avions préservé une plus grande partie de la littérature hellénistique, nous connâ̂trions mieux la critique esthétique de cette période. En fait, on trouve dans Polybius des opinions qui nous font supposer qu'une théorie esthétique a également existé. On peut en dire autant des Néoptolèmes de Parion, que nous connaissons par l'Art poétique d'Horace. Cependant, Filodemos est le premier auteur que nous connaissons assez bien pour pouvoir affirmer qu'il argumente à partir d'une théorie esthétique élaborée. Et l'admirable opuscule 


\begin{abstract}
Du Sublime, un fragment du milieu du premier siècle après J.-C., est presque le seul ouvrage de l'Antiquité qui repose sur une certaine conception esthétique. Dans ces conditions, Reyes adopte à juste titre 'un critère de tolérance'. [Dans les 143 premières pages], son but [...] a été de montrer 'comment le phénomène de la critique littéraire est né et a évolué'. Il sera difficile de soutenir qu'il a pleinement atteint son objectif. Son aperçu ressemble davantage au volumineux livre de Gomperez sur les penseurs grecs qu'à une analyse stricte de l'évolution de la critique littéraire (Düring 1955, 26-27).
\end{abstract}

Les philosophes et philologues mexicains, en général, soutiennent la position de Jaeger ou celle de Düring. Par exemple, José Gaos, proche des opinions de Jaeger, a déclaré: « Avec ce travail, Alfonso Reyes a entamé un nouveau parcours de son œuvre et de sa vie : celui de la réflexion, non plus occasionnelle et marginale, mais thématique et systématique sur son métier » (Gaos 1955, 500). Pour sa part, Carlos Montemayor, plus proche des conceptions de Düring, a déclaré: « Reyes a négligé ce qui était le plus important chez Aristophane: la critique littéraire directe basée sur l'écrivain lui-même. Pourquoi ne l'a-t-il pas abordé? Peut-être parce que la lecture critique des Hellénistes de son temps pesait plus lourdement sur lui que la lecture directe et naturelle du texte grec lui-même » (Montemayor 1989, 154) ${ }^{3}$.

Dans le second domaine, parmi ceux qui regrettent le manque de problématiques mexicaines, figurent surtout de jeunes écrivains (narrateurs, poètes, romanciers) qui demandent un plus grand engagement éthique des intellectuels face aux conjonctures historiques de l'époque. Pour beaucoup d'entre eux, les préoccupations grecques d'Alfonso Reyes représentent une façon de prendre la

3 L'une des questions les plus controversées, par rapport au type d'hellénisme pratiqué par Alfonso Reyes, est sa connaissance limitée de la langue grecque. De nombreux spécialistes refusent de le considérer comme un helléniste en raison de cette méconnaissance. Antonio Alatorre, qui l'a accompagné pendant plusieurs années au Colegio de México, a déclaré: « Don Alfonso connaissait le grec comme je connaissais le russe: il lisait les lettres et comprenait certains mots isolés, mais c'était tout [...]. Et d'ailleurs, il a toujours avoué que sa traduction d'Homère était une traduction des traductions françaises et anglaises » (Alatorre 1974, 22). Antonio García Robles, dans le même sens, a également regretté que le plus grand introducteur de la Grèce classique au Mexique n'ait pas les outils de base du langage de l'Antiquité (Montemayor 1989, 12). Reyes lui-même l'a bien précisé lorsque, dans sa traduction de l'Iliade, il a déclaré: « Je ne lis pas la langue d'Homère; je la déchiffre à peine " (Reyes 1968 [1951], 91). Bien sûr, ce fait nous amène à nous interroger sur le sens de ses incursions dans le monde antique. Il était clair que Reyes n'agissait pas en tant que philologue dans le monde classique de la Grèce. Lui qui avait pratiqué cette discipline au Centro de Estudios Históricos de Madrid, aux côtés de Ramón Menéndez Pidal et d'Américo Castro, savait parfaitement quelles étaient les exigences minimales pour travailler en tant que spécialiste d'une tradition littéraire. Reyes, à mon avis, a toujours abordé l'antiquité classique comme un essayiste, comme un intellectuel qui propose des solutions, en s'aventurant dans le monde antique, pour le présent. Reyes a trouvé dans ce monde antique des éléments pour mieux comprendre son présent. 
fuite ou d'échapper aux conflits de son temps. Pour José Revueltas, par exemple, le panorama littéraire du Mexique vers 1943 peut être divisé en plusieurs groupes : les hellénistes, les occidentalisés, les révolutionnaires et les marxistes. Parmi les premiers, bien sûr, se trouvait Alfonso Reyes:

Les Hellénistes aspirent à se placer " au-dessus du désordre ", à la tête duquel se trouve Don Alfonso Reyes en tant que leader et prototype («Souvenez-vous », a dit Don Alfonso Reyes à un ami de province, dans une lettre que le destinataire a eu l'indiscrétion de me lire, « souvenez-vous que les penseurs grecs étaient payés par la municipalité; ne vous plaignez pas. Nous allons dire notre petite vérité, malgré tout ». La citation n'est pas textuelle mais reflète très précisément la pensée de Reyes) Ces hellénistes ont même pu comprendre les problèmes du Mexique; mais ils veulent qu'on les laisse tranquilles, qu'on leur permette avec cinq ou six cents pesos de salaire - de forger les plus hautes méditations. Ils ne risquent que de dire peu et lâchement et mal, sans faire de mal à personne, sans blesser, surtout, celui qui donne les six cents pesos (Revueltas 1943, 3).

Face à ces deux univers de réception, j’aimerais étayer une troisième possibilité de lecture. Il me semble que La critique à l'âge athénien n'est pas un travail philologique au sens le plus strict, mais un essai d'interprétation qui met en actualité, au sens de Walter Benjamin, ou rend contemporain, selon les termes de Giorgio Agamben (2011, 17-30) un héritage culturel, pour formuler une série d'inquiétudes sur la formation de la Polis et sur le rôle de l'intellectuel dans un moment de crise profonde, comme l'a été la Seconde Guerre mondiale, vécue depuis un pays en marge du conflit armé. Les pages suivantes se situent dans cette troisième voie d'interprétation qui implique une lecture politique de ce travail sur la Grèce ${ }^{4}$.

4 En faisant une lecture politique du texte de Reyes, je n'ai pas l'intention de révéler la position ou la préférence idéologique de l'auteur, mais plutôt d'enquêter sur le partage du sensible que le texte de Reyes lui-même propose dans la configuration artistique de son écriture (Rancière 2000). En ce sens, Ignacio Sánchez Prado (2008) et Robert T. Conn (2002) ont publié des ouvrages fondamentaux pour entreprendre cette lecture politique de quelques essais d'Alfonso Reyes. Un antécédent à cette perspective d'analyse se trouve dans les travaux de Rafael Gutiérrez Girardot qui, en 1990, a déclaré: « [dans ses travaux sur la Grèce] Reyes n’a pas voulu se distinguer comme philologue [...]. Reyes voulait susciter, présenter des exemples d'humanité et surtout répondre aux besoins essentiels qui avaient été imposés à l'intelligence américaine par l'entrée tardive de l'Amérique dans l'histoire de l'Occident. [... Le faible poids de la tradition, c'est-à-dire de la philologie classique, a permis à Reyes de créer une image de la Grèce qui, en plus d'être exemplaire, était proche de celle que Nietzsche avait esquissée dans L'origine de la tragédie dans l'esprit de la musique (1872). C'est une Grèce esthétique qui, comme l'exigeait Nietzsche, s'est concentrée sur l'ensemble et non, comme le faisait la philologie classique, sur les détails. Mais cette Grèce esthétique n’a pas cessé d'être exemplairement politique » (Gutiérrez 1990, 107-108). 


\section{Modélisations citoyennes en temps de guerre}

Même si en apparence l'ouvrage de Reyes ne concerne en rien la situation politique de l'époque, le volume contient de nombreux indices qui révèlent une profonde préoccupation se rapportant aux évènements d'alors. En principe, le désir de questionner la notion et l'origine de « la critique » montre déjà une inquiétude par rapport à l'actualité. Dans un essai de la fin de l'année 1939, Alfonso Reyes signale: « Le mal de notre époque [...] réside dans une certaine distorsion du sens critique, dans une certaine érosion des résistances aux tromperies » (Reyes 1960, 250). Face à un scénario contemporain où prolifèrent les discours mensongers, irrationnels et belliqueux, Reyes propose dans son livre de 1941 une sorte de prophylaxie du langage: " la netteté de la forme », due aux activités critiques, mène « à l'épuration de la pensée » (Reyes 1941, 200). L'urgence dans la recherche d'une hygiène du discours n'est pas la seule préoccupation sur l'actualité. En écrivant le livre, Reyes sublime ses angoisses, son malaise et ses craintes.

Dès le premier paragraphe, l'essayiste déploie des figures et des termes qui renvoient à un univers combatif. L'écrivain souhaite former l'histoire de la critique mais pendant son trajet, en reconstruisant la culture de l'Athènes classique, il recrée des images du guerrier, de l'armée, du combattant; il décrit des scénarios de violence, de destruction, de guerre; tout cet univers se veut d'être conjuré de citations qui prêchent la concorde et la paix. Aristophane, dit Reyes, charge contre les puissants, mais surtout contre « le monstre épouvantable de la guerre » (Reyes 1941, 147); Isocrate prône l'union de la « famille grecque ravagée par les guerres intestines » (Reyes 1941, 190); Socrate, le soldat, découvre sa mission philosophique au milieu d'une bataille (Reyes 1941, 94-95). Tout cela n'est pas gratuit. En réalité, Reyes est vraiment inquiet du déroulement du conflit européen. En s'interrogeant sur l'origine et la fonction de la critique, il questionne également le rôle des intellectuels dans un moment de conflit. Ces inquiétudes apparaissent dans les annotations de son journal. Le 10 mai 1940, il écrit: «L'Allemagne envahit la Hollande, le Luxembourg, la Belgique et bombarde Londres [...]. Angoissé par les évènements mondiaux » (Reyes 2018, 174-175). Le 14 juin, il témoigne: "Les nazis à Paris, deuil du genre humain » (Reyes 2018, 181). Quelques jours plus tard, il énonce: «Enfermé, enivré de travail, mais très angoissé... Les États-Unis - et même le Mexique - vont bientôt devoir se militariser pour une nouvelle guerre » (Reyes 2018, 181). Le 13 octobre, en pleine rédaction de La critique à l'âge athénien, il commente: « Mauvaise nuit, je pense à [...] la situation du pays et la situation du monde. Je ne me fais aucune illusion sur rien. Je continue d'écrire sur mes grecs » (Reyes 2018, 201). Des nouvelles sur la guerre ont dû parvenir à ses oreilles directement et de façon personnelle. À partir de 1939, des milliers de réfugiés européens 
fuyant la guerre commencent à arriver au Mexique. Des républicains espagnols vaincus par Franco ; des communistes allemands persécutés par Hitler ; des antifascistes italiens exilés par Mussolini; des militants français expulsés par Vichy; tous sont accueillis au Mexique, d'abord par le gouvernement de Cárdenas puis par celui de Ávila Camacho. Reyes rencontre directement beaucoup d'entre eux, surtout parmi les groupes intellectuels. Si l'on tient compte de cet univers de migrations, d'exils et de déracinements, la lecture de certains fragments de La critique à l'âge athénien est révélatrice:

La guerre de Troie est un échec. [...] Nous savons seulement que les héros grecs entreprennent un retour lamentable, une odyssée laborieuse, pour finalement retrouver leurs foyers détruits et leurs terres réduites à l'anarchie [...]. Pour les gens dont les cultes et les coutumes sont désintégrés par les émigrations et les guerres, le progrès consiste à rendre la cohabitation entre les hommes plus paisible et possible; c'est-à-dire, à augmenter la confiance dans les pactes sociaux et assurer leur continuité. L'État est un produit artificiel. Il faut l'aider à percer avec un propos conscient [...]. La critique, tout comme d'autres activités de l'esprit, se soumettra à cette consigne qui est une aspiration générale (Reyes 1941, 33).

Dans cette dernière phrase, Reyes met directement en relation le travail des critiques athéniens avec leur rôle dans la construction de l'état. Les intellectuels du $\mathrm{XX}^{\mathrm{e}}$ siècle se doivent d'assurer cette même fonction : "La critique à l'âge athénien révèle une ambition institutionnelle. Les critiques de ce temps-là [...] sont déterminés à construire et sauvegarder la Polis, a l'instar de toute la pensée grecque de l'époque » (Reyes 1941, 32). « Construire et sauvegarder la Polis », deux actions que Reyes semble alors accomplir à la tête de El Colegio de México, en charge du Fondo de Cultura Económica ou dans ses cours au Colegio Nacional.

Cependant, un fragment de La critique à l'âge athénien est particulièrement révélateur. Pratiquement à la fin de l'ouvrage, Reyes inclut un chapitre intitulé « Un athénien quelconque ». Il y dresse le portrait imaginaire d'un habitant présumé d'Athènes au IV ${ }^{\mathrm{e}}$ siècle. Reyes imagine la vie quotidienne de ce personnage : son foyer décent et modeste, sans parures ni luxe; sa vie familiale simple; sa magnanimité envers les aides et sa patience envers les voisins; il dialogue avec ses enfants, puisque « le dialogue est la discipline de la connaissance »; gère ses finances en toute discrétion; respecte les institutions; il n'est ni indiscret ni hautain.

Sa vie de famille est simple et conforme à l'ancienne coutume: avec sa femme, il est libéral et un peu distant; avec ses enfants, ponctuel et soigneux; avec ses serviteurs, magnanime; avec ses voisins, patient. [...]. Chacun sait qu'il vit sans difficultés et sans abondance; qu'il gère ses finances avec discrétion; qu'il ne prête qu'en sûreté; qu'il n'avance jamais d'intérêts avant les mensualités [...]. Il respecte les institutions [...]. Il essaie de ne pas trop attirer l'attention sur lui dans la rue ou dans les réunions [...]. Il ne tombe pas dans la sottise du 
peuple, qui s'arrête pour regarder passer le bœuf, la chèvre, l'âne, comme s'il ne les avait jamais vus auparavant. Il ne s'immisce pas dans les écoles et les gymnases pour prendre le temps des garçons et des enseignants avec ses conversations gênantes. Il ne se mêle pas des querelles des étrangers [...]. Il ne prétend pas savoir ce qu'il ne sait pas (Reyes 1941, 350-355).

Chacun des éléments que souligne Reyes dans sa description tisse une trame éthique: les comportements austères du personnage qui cherche l'équilibre des émotions; il favorise la résolution des conflits; il est réservé et prudent; vit avec pondération et équilibre. La pratique du juste milieu (le livre est composé de 555 paragraphes numérotés) est considérée comme une vertu. Dans cette histoire, Reyes présente la figure d'un homme comme modèle de comportement. Dans un essai ultérieur à ce livre, en traitant des discours irrationnels et du nazisme, Reyes déclare que nous ne pouvons opposer la raison à l'irrationalisme, mais la pratique de la vertu comme modèle de comportement:

La raison doit reconnaître les forces irrationnelles qui affectent si profondément la pensée et le comportement des hommes [...] pour lutter contre ces forces, nous devons comprendre leur dynamique, et nous devons nous rappeler que nous ne les combattons pas avec des arguments (parce que cela serait inutile) [...] mais en appliquant patiemment cette méthode ou frein social qu'Aristote appelait éthisme: exercice conscient et réitération d'une vertu pour l'acquérir enfin, un remède pragmatique (Reyes 1968 [1952], 358).

C'est pour cela que l'élaboration fictionnelle d'un habitant de la polis grecque à la fin de son ouvrage sur la critique athénienne peut être lue comme la formalisation artistique d'un projet de modélisation éthique à l'intention du citoyen.

Il est clair que cette modélisation concerne directement l'espace et la polis mexicaine, mais pas seulement. D'une certaine façon, son livre formalise également les inquiétudes d'un groupe d'intellectuels européens et américains qui, auparavant, pendant et après la Seconde Guerre Mondiale, ont tenté de concrétiser un projet de formation de l'être humain face au scénario d'une profonde crise armée. Dans le contexte des activités et des inquiétudes de ce groupe, le projet d'Alfonso Reyes d'un humanisme universaliste qui s'assume comme héritier de la Grèce classique peut être compris avec plus d'exactitude.

\section{Les politiques de l'humanisme}

Mark Greif (2015), dans un ouvrage récent sur le concept de l'homme dans la fiction nord-américaine, assure que la première moitié du XX $\mathrm{XX}^{\mathrm{e}}$ siècle en Occident se caractérise par une crise du concept de l'«humain». De nombreuses recherches sur l'humanité et l'humanisme se reflètent dans les ouvrages de phi- 
losophes, psychologues, historiens et écrivains. Cette prolifération de recherches sur l'« homme » fait partie du résultat d'une crise déclenchée par l'ambiance conflictuelle qui avait imprégné les premières décennies du siècle. Devant la subversion des valeurs politiques, économiques, sociales et culturelles, la réaction immédiate est de s'interroger sur le sens et la signification de l'« homme ». C'est ainsi que surgissent diverses propositions de modélisation de l'être humain: celle du fascisme, celle du nazisme, celle du stalinisme, celle du libéralisme. Quasiment tous les mouvements politiques évoquent la formation d'un « homme nouveau » (Greif 2015, 3-99).

Parmi les nombreux groupes d'intellectuels de cette époque, préoccupés par l'idée de formation de l'être humain, je ne m'arrêterai que sur un seul. Il concerne directement le projet d'humanisme grec et universaliste d'Alfonso Reyes. Je fais référence à l'ensemble d'écrivains et d'intellectuels qui, à partir de 1932, s'organise au sein de l'Institut International de Coopération Intellectuelle - prédécesseur direct de l'UNESCO et dépendant à ce moment-là de la Société des Nations. Paul Valéry et Henri Focillon sont les deux figures clé aux débuts de l'organisme. Tous deux entreprennent de convoquer les intelligences mondiales afin de faciliter les échanges intellectuels. L'objectif est de réunir « les hommes les plus capables d'éclairer la conscience universelle et de s'éclairer mutuellement à une heure particulièrement grave de la vie du monde » (Valéry et Focillon 1933, 13). Le mexicain Alfonso Reyes est témoin de plusieurs moments. En 1932, il répond à l'appel de Valéry pour fonder la Société des esprits (Ugalde 2017). En 1936, il participe au $6^{\mathrm{e}}$ congrès de l'Institut International de Coopération Intellectuelle mené à Buenos Aires (Colombi 2011). En 1941, il préside les activités de cette institution à La Havane (Pita 2014). En 1943, il fait office de président du Bureau à New York, alors que l'institut était déjà exilé.

Toutes les rencontres, colloques et conversations de cet organisme expriment le désir de rétablir le sens de l'humain et de tracer les activités de la littérature et des intellectuels dans la construction d'un nouvel humanisme. Il suffit de revoir les titres de ces évènements pour se rendre compte des dimensions pédagogiques de cet effort, notamment le colloque réalisé à Budapest en juillet 1935 qui porte le titre suggestif de "Vers un nouvel humanisme ». Plusieurs notions de ce terme dialoguent lors de ce congrès: celle des philologues classiques qui prétendent renforcer l'enseignement des langues classiques; celle de Paul Valéry qui fait une série d'annotations sur l'humanisme pur; celle de Thomas Mann qui, face au scénario de la montée du nazisme, demande un humanisme militant avec une incidence politique directe (Mann 1936). Quelques mois après la rencontre à Budapest, l'Institut organise un autre colloque à Buenos Aires. Le thème proposé: les relations intellectuelles entre l'Europe et l'Amérique. Deux représentants de chacun des continents sont chargés d'ouvrir le colloque. Pour l'Europe, George 
Duhamel fait le discours d'inauguration; pour l'Amérique, ce sera Alfonso Reyes. L'écrivain mexicain y lit un essai clé dans son œuvre littéraire: Notas sobre la inteligencia americana (Reyes 1936). Ce texte répond aux inquiétudes humanistes exposées dans l'IICI.

Reyes soutient dans ce texte que les intellectuels dans l'Amérique hispanique ont toujours dû mener leurs activités sur la place publique. C’est pour cette raison que l'intelligence américaine ne peut penser les intellectuels purs - une allusion claire à Valéry et à Julien Benda -, mais les intellectuels engagés avec leur époque et leur société. Les intellectuels hispano-américains avaient dû associer leurs préoccupations sur le savoir avec la construction des sociétés nationales. Ils représentent la plume et la place publique, l'écriture et la politique. Cette condition désavantageuse à d'autres moments, semble être idéale lors du scénario conflictuel vécu à l'époque. En plus de leur caractère public, les intellectuels américains incarnent l'idée de synthèse de tout l'héritage d'Occident (Reyes 1936, 1418). Quelques années plus tard, Reyes élabore La critique à l'âge athénien sous ces deux principes. Il y concrétise l'idée d'une intelligence qui organise la synthèse d'un héritage culturel depuis la place publique.

Dans ce contexte, l'ouvrage d'Alfonso Reyes répond alors à un double horizon politique et discursif: d'un côté, il satisfait aux besoins de l'état postrévolutionnaire mexicain, en proposant des formes de modélisation citoyenne à un moment de consolidation institutionnelle; d'un autre côté, il répond aux inquiétudes exposées par les intellectuels regroupés au sein de l'IICI, en atténuant les angoisses face à la guerre et en proposant le sauvetage - le processus d'actualiser - d'un héritage culturel. Pour l'état Mexicain et pour l'IICI, il est nécessaire de montrer des façons d'être, d'agir et de dire qui soient propices à la consolidation d'une nouvelle polis, locale et globale. Reyes sait que son œuvre littéraire et essayiste se situe à la croisée de ces modèles de comportement. Lors du discours de remise du premier Prix National de Littérature, il assure: « le problème de la cohabitation de l'homme avec l'homme, le problème humain par excellence sur terre, soit le problème politique, en plus d'exiger des procédures d'application courte et immédiate, ne connaît qu'un seul remède à long terme [...] qui, en résumé, est appelé culture » (Reyes 1945). Une culture qui, évidemment pour Reyes, équivaut à la Grèce classique. Peu après, dans une interview, Alfonso Reyes a souligné le sens politique et formateur d'offrir une vision de l'humanisme classique: « Je crois que le sens du véritable humanisme consiste à réintégrer la théorie et la technique dans la politique. C'est-à-dire, dans l'idéal humain de la meilleure et de la plus juste coexistence de l'homme avec l'homme [...]. Les doctrines des humanistes doivent être ressuscitées afin de les ramener en harmonie avec les fins de l'humanité » (Reyes 1946). Cette modélisation à partir de l'idéal grec implique bien entendu de nombreuses exclusions ; les plus évidentes : celle de genre et celle 
des cultures non occidentales. Les femmes et les populations autochtones mexicaines sont une présence très conflictuelle dans l'œuvre littéraire de l'humaniste Alfonso Reyes.

Quelques jours à peine avant la remise du prix à Alfonso Reyes, en cette même année 1945, les activités de l'IICI portent leurs fruits et l'UNESCO est fondée. L'objectif de cette institution est de contribuer à la paix et à la sécurité mondiale à travers l'éducation, la science et la culture. Le président du Mexique, Manuel Ávila Camacho, lors de la remise du premier Prix National de Littérature, fait un discours qui met subtilement en relation les efforts de l'état mexicain pour consolider la polis littéraire du pays avec la création récente de l'UNESCO. De manière indirecte, le président du Mexique situe les efforts et l'œuvre d’Alfonso Reyes dans un horizon global:

Il est extrêmement révélateur qu'aussitôt le fracas de la lutte armée ayant pris fin, quarantetrois pays se soient unis pour déclarer leur résolution d'affirmer la sécurité collective des nations sur l'indépendance de la pensée, à travers l'éducation, la science et la culture. Étant donné que c'est dans l'esprit de l'être humain que doivent s'ériger primordialement les bastions magnifiques de la paix, ces pays exaltent une volonté de concorde que le Mexique a soutenu sans faiblir, à travers les sacrifices et les tourmentes [...] orientée et régie par la justice, la culture sera toujours la défense la plus généreuse et l'argument le plus convaincant d'un peuple dans son dialogue avec le monde civilisé (Ávila Camacho 1945, 2).

De cette manière, avec la remise du premier Prix National de Littérature au Mexique, plusieurs inquiétudes et projets de toute une communauté intellectuelle sont symboliquement rattachés. L'ouvrage d'Alfonso Reyes, La critique à l'âge athénien, n'est qu'un symptôme et une réponse aux angoisses, contradictions et recherches que de nombreux écrivains et intellectuels ont tenté de résoudre en ayant recours à un passé et à un héritage de la culture - en l'occurrence la Grèce comme solution universaliste à un horizon conflictuel dominant.

\section{Références bibliographiques}

Agamben, Giorgio. «¿Qué es lo contemporáneo?». Desnudez. Trad. Mercedes Ruvituso y Maria Teresa D’Meza. Buenos Aires: Adriana Hidalgo, 2011, 17-29.

Alatorre, Antonio. «Alfonso Reyes: pequeña crónica desmitificante». Diálogos. Artes/Letras/ Ciencias Humanas 58 (1974): 20-22.

Ávila Camacho, Manuel. «Discurso de entrega del Premio Nacional de Literatura». El Nacional 2 (21 décembre 1945).

Bhabha, Homi. Nuevas minorías, nuevos derechos. Trad. Mariano Siskind. Buenos Aires: Siglo XXI, 2013.

Colombi, Beatriz. «Alfonso Reyes y las "Notas sobre la inteligencia americana” una lectura en red». Cuadernos del CILHA 12.14 (2011): 109-123. 
Conn, Robert T. The Politics of Philology: Alfonso Reyes and the Invention of the Latin America Literary Tradition. Lewisburg, London: Bucknell University/Associated University, 2002.

Díaz Arciniega, Víctor. Querella por la cultura revolucionaria (1925). México: Fondo de Cultura Económica, 1989.

-. Premio Nacional de Ciencias y Artes (1945-1990). México: Fondo de Cultura Económica, 1991.

Düring, Ingemar. Alfonso Reyes: helenista. Madrid: Ínsula, 1955.

Garciadiego, Javier. Alfonso Reyes, breve biografía. México: Planeta, 2009.

Gaos, José. «La crítica en la edad ateniense». Páginas sobre Alfonso Reyes vol. 1, segunda parte. Éd. Alfonso Rangel Guerra. México: El Colegio Nacional, 1955, 494-503.

Greif, Mark. The Age of the Crisis of Man. Thought and Fiction in America, 1933-1973. Princeton, NJ: Princeton University Press, 2015.

Gutiérrez Girardot, Rafael. «La concepción de Hispanoamérica de Alfonso Reyes (1889-1959)». Revista de Occidente 106 (1990): 107-108.

Jaeger, Werner. Paideia: Die Formung des griechischen Menschen. Berlin, Leipzig: De Gruyter, 1933.

Mann, Thomas. «Participation». Entretiens 6. Vers un nouvel humanisme. Paris: Institut International de Coopération Intellectuelle, Societé des Nations, 1936, 53-55.

Montemayor, Carlos. «El helenismo de Alfonso Reyes». Vuelta 154 (1989): 12-16.

Niemeyer, Eberhardt Victor. El general Bernardo Reyes. Monterrey: Gobierno de Nuevo León/ Universidad de Nuevo León, 1966.

Paz, Octavio. «El ogro filantrópico». Vuelta 21 (1978) : 38-44.

Pérez Montfort, Ricardo. Lázaro Cárdenas, un mexicano del siglo XX. México: Debate, 2019.

Pita González, Alejandra. Educar para la paz. México y la Cooperación Intelectual Internacional 1922-1948. México: Secretaria de Relaciones Exteriores/Universidad de Colima, 2014.

Quintanilla, Susana. «Dioniso en México o cómo leyeron nuestros clásicos a los clásicos griegos». Historia Mexicana 51 (2002): 619-663.

-. Nosotros La juventud del Ateneo de México. México: Tusquets, 2008.

Rancière, Jacques. Le partage du sensible: Esthétique et politique. Paris: La Fabrique, 2000.

Rangel Guerra, Alfonso. Las ideas literarias de Alfonso Reyes. México: El Colegio de México, 1989.

Reyes, Alfonso. «Exposé introductif de M. Alfonso Reyes. [Peu de temps après, ce texte portera le titre: « Notas sobre la inteligencia americana »]». Entretiens 7. Europe - Amérique Latine. Paris: Institut International de Coopération Intellectuelle, 1936, 12-19.

-. La crítica en la edad ateniense. México: El Colegio de México, 1941.

-. «Discurso de recepción del Premio Nacional de Literatura», 1945. Archivo Histórico de la Capilla Alfonsina en la Ciudad de México, fol. AR-MAN-03344.

-. «Interviu con Alfonso Reyes para la revista Así», 1946. Archivo Histórico de la Capilla Alfonsina en la Ciudad de México, fol. AR-MAN-03435.

-. Tentativas y orientaciones. Obras completas vol. XI. México: Fondo de Cultura Económica, 1960.

-. «La llíada de Homero». Obras completas vol. XIX. México: Fondo de Cultura Económica, 1968 [1951].

-. «Las agonías de la razón». Obras completas vol. XIX. México: Fondo de Cultura Económica, 1968 [1952].

-. Diarios V (1939-1945). Éd. Javier Garciadiego. México: Fondo de Cultura Económica, 2018.

Revueltas, José. «Réplica sobre la novela: el cascabel al gato». El Popular 3 (23 mai 1943). 
Sánchez Prado, Ignacio. «Alfonso Reyes y la crítica clásica. Notas para una genealogía». Revista Anthropos. Huellas del Conocimiento 221 (2008): 96-107.

Sheridan, Guillermo. México en 1932: la polémica nacionalista. México: Fondo de Cultura Económica, 1999.

Sisikind, Mariano. Deseos cosmopolitas. Modernidad global y literatura mundial en América Latina. Trad. Lilia Mosconi. Buenos Aires: Fondo de Cultura Económica, 2016.

Ugalde Quintana, Sergio. Éd. Un amigo en tierras lejanas: correspondencia Alfonso Reyes/ Werner Jaeger (1942-1958). México: El Colegio de México, 2009.

-. «Das Vermögen der Intellektuellen: Paul Valéry und Alfonso Reyes am Internationalen Institut für geistige Zusammenarbeit». Paul Valéry: Für eine Epistemologie der Potentialität. Éds Pablo Valdivia Orozco et Andrea Allerkamp. Heidelberg: Winter Universitätsverlag, 2017, 253-268.

-. «Alfonso Reyes lee a Nietzsche: cultura clásica y ethos agonista». Nueva Revista de Filología Hispánica 67 (2019) : 131-153.

- et Ottmar Ette. Éds. Políticas y estrategias de la crítica II. Ideología, historia y actores de los estudios literarios. Frankfurt am Main, Madrid: Vervuert Iberoamericana. à paraître.

Valéry, Paul et Henri Focillon. «Introduction». Correpondance 1. Pour une société des Esprits. Lettres de Hewnri Focillon, Salvador de Madariaga, Gilbert Murray, Miguel Ozorio de Almeida, Alfonso Reyes, Tsaï Yuan Peï. Paris: Institut International de Coopération Intellectuelle, Societé des Nations, 1933.

Vasconcelos, José et al. «Informe de la comisión administradora del premio Nacional de Literatura, para el año de 1945», 1945. Archivo Histórico de la Capilla Alfonsina en la Ciudad de México, fol. AR-MAN-05450. 